\title{
Approximate dissipative Hamiltonian realization and construction of local Lyapunov functions
}

\author{
Yuzhen Wang ${ }^{\mathrm{a}, *}$, Daizhan Cheng ${ }^{\mathrm{b}}$, S.S. Ge $\mathrm{Ge}^{\mathrm{c}}$ \\ ${ }^{a}$ School of Control Science and Engineering, Shandong University, Jinan 250061, China \\ ${ }^{\mathrm{b}}$ Institute of Systems Science, Chinese Academy of Sciences, Beijing 100080, China \\ ${ }^{\mathrm{c}}$ Department of Electrical and Computer Engineering, the National University of Singapore, Singapore 117576, Singapore
}

Received 4 August 2003; received in revised form 14 January 2005; accepted 25 August 2006

Available online 29 September 2006

\begin{abstract}
The key in applying energy-based control approach is to be able to express the system under consideration as a dissipative Hamiltonian system, i.e., to obtain Dissipative Hamiltonian Realization (DHR) for the system. In general, the precise DHR form is hard to obtain for nonlinear dynamic systems. When a precise DHR does not exist for a dynamic system or such a precise realization is difficulty to obtain, it is necessary to consider its approximate realization. This paper investigates approximate DHR and construction of local Lyapunov functions for time-invariant nonlinear systems. It is shown that every nonlinear affine system has an approximate DHR if linearization of the system is controllable. Based on the diagonal normal form of nonlinear dynamic systems, a new algorithm is established for the approximate DHR. Finally, we present the concept of $k$ th degree approximate Lyapunov function, and provide a method to construct such a Lyapunov function. Example studies show that the methodology presented in this paper is very effective.
\end{abstract}

(C) 2006 Elsevier B.V. All rights reserved.

Keywords: $k$ th degree approximate DHR; $k$ th degree approximate Lyapunov function; Diagonal normal form; Algorithm

\section{Introduction}

Energy-based control and stability analysis have been extensively studied for a wide range of physical systems, which include robotic manipulators [10,15], surface vehicles [11], space crafts [26], mechanical systems [27,2], and electrical systems [20,9], etc. While much research work in the area of robotics leads to a good understanding of this approach, its recent successful applications to power systems require new problem formulations and new insights into this approach $[14,28]$. In recent years, port-controlled Hamiltonian systems $[32,31,18]$ have been well investigated in a series of works $[19,7,17,21-23,12,13]$. A constructive procedure was proposed in [19] to modify the Hamiltonian function of forced

\footnotetext{
it Supported by the National Natural Science Foundation of China [G60474001], and the Research Fund of Doctoral Program of Chinese Higher Education [G20040422059].

* Corresponding author. Tel.: +865318392515; fax: +865318392205 .

E-mail addresses: wangyuzhen@tsinghua.org.cn (Y. Wang), dcheng @iss03.iss.ac.cn (D. Cheng), elegesz@nus.edu.sg (S.S. Ge).
}

Hamiltonian systems with dissipation to generate a Lyapunov function for non-zero equilibria. In [7], through the fundamental concept of a generalized Dirac structure [6] defined on the space of energy variables, it was shown that a power-conserving interconnection of port-controlled generalized Hamiltonian systems leads to an implicit generalized Hamiltonian system, and a power-conserving partial interconnection to an implicit protcontrolled Hamiltonian system. The Hamiltonian function, the sum of potential energy (excluding gravitational potential energy) and kinetic energy in physical systems, is a good candidate of Lyapunov functions for many physical systems, which makes it very easy for stability analysis and control design for the systems. Up to now, the port-controlled Hamiltonian approach has been used in various control problems [17,9,23]. In particular, it has been successfully applied to the control of power systems in a series of works $[3,24,14,28]$.

In order to apply the energy-based approach, it is important to be able to express the system under consideration as a dissipative Hamiltonian system, i.e., to obtain the dissipative Hamiltonian realization (DHR) for the system $[4,29]$. In general, 
to obtain the DHR, we first need to express the system as a generalized Hamiltonian system, which is the so-called generalized Hamiltonian realization (GHR) [4,29], and then eliminate the non-dissipative part of the obtained GHR by a suitable state feedback. GHR/DHR is a very difficult issue for general nonlinear systems, since it involves solving certain partial differential equations [30] which is usually very difficult to solve. For time-invariant nonlinear systems, the GHR/DHR problem has been studied in some recent works [4,29,23,25,30]. Indeed, there are many results on GHR/DHR of nonlinear systems. However, most of the results only establish sufficient conditions for the existence of Hamiltonian realization, and cannot provide any applicable algorithm to compute an explicit GHR or DHR for general nonlinear systems. In [29,23], it was shown that the input-state part of every asymptotically stable system can be expressed as a port-controlled Hamiltonian system with dissipation. But, these papers completed their proofs by using the inverse Lyapunov theorem [16], i.e., under the assumption that there exists a Lyapunov function for every asymptotically stable system. As it is well known, Lyapunov functions are hard to obtain for general nonlinear systems, and thus the results presented in $[29,23]$ are hard to use to construct an explicit GHR or DHR form for general asymptotically stable systems. This is the very fact that motivates us to investigate the method of constructing explicit DHRs.

In fact, without the explicit DHR form, it is impossible to handle the control design problem based on Hamiltonian framework, even if it is known that a DHR form exists for the system under consideration. As mentioned earlier, DHR is a very difficult issue for nonlinear systems. In general, the precise form of Hamiltonian realization is very hard to obtain for nonlinear systems. This is because, on one hand, there is no precise DHR for some systems and, on the other hand, it is impossible to construct it analytically for many systems, even though it is known that the DHR exists for the systems. When a precise DHR does not exist for a dynamic system or such a precise realization is difficult to obtain, we may consider its approximate realization. Its approximate realization up to certain degree may be enough for practical control problems. Thereby, it is necessary to formulate approximate Hamiltonian realization. In [4], the Carleman linearization procedure was applied to study the approximate GHR problem of nonlinear systems, and an algorithm was proposed for the GHR. However, the algorithm is not applicable to the approximate DHR, which is really the case we concern about. To the authors' best knowledge, there are few results on the approximate DHR of nonlinear systems thus far.

In this paper, we investigate the approximate DHR problem of time-invariant nonlinear systems, and present a new algorithm for the approximate DHR. The concept of $k$ th degree approximate DHR is proposed first, and then, based on the diagonal normal form of dynamic systems [5], it is shown that every nonlinear affine system has a $k$ th degree approximate DHR if linearization of the system is controllable, where $k$ is an arbitrary natural number. Finally, we give a new algorithm for the $k$ th degree approximate DHR of time-invariant nonlinear systems. The new algorithm, which is first presented in this paper, is established through a comparatively standard procedure.
This can bring some facilities to calculation of the explicit realization. The new algorithm is very easy and practical to apply, and has the following advantages in handling approximate DHR and correlative control problems:

(1) The condition required in the new algorithm is less demanding and can be satisfied by most practical systems. Therefore, the proposed algorithm will have a wide range of applications.

(2) It can give approximate DHR up to any degree for nonlinear systems, which can make the approximation meet practical requirements for most nonlinear systems.

(3) Based on the new algorithm, it can be shown that every polynomial system has a precise DHR, and moreover, the precise DHR can be explicitly given by the new algorithm (See Corollary 3.11 for details).

(4) The new algorithm not only can yield an approximate DHR for the system whose linearization is controllable, but also can provide a useful method to construct local Lyapunov function for the system.

Another aim of this paper is to apply the new algorithm to investigate construction of local Lyapunov functions. Lyapunov functions play a key role in stability analysis and control design. As mentioned earlier, such a function is very hard to obtain for general nonlinear systems. Thereby, it should be a feasible way to construct an approximate Lyapunov function for the systems whose Lyapunov function cannot be obtained by using the existing methods. In this paper, we present the concept of $k$ th degree approximate Lyapunov function, which is a kind of local Lyapunov function, and then, based on the approximate DHR algorithm obtained in this paper, we provide a method of constructing the $k$ th degree approximate Lyapunov function. The presented example shows that the algorithm proposed in this paper is very feasible.

The rest of the paper is organized as follows: Section 2 gives some preliminary results. In Section 3, we deal with the $k$ th degree approximate DHR and give the new algorithm for the approximate DHR. Section 4 investigates the construction of the $k$ th degree approximate Lyapunov function. Section 5 presents an illustrative example, which is followed by the conclusion in Section 6.

\section{Preliminaries}

To facilitate the analysis, some fundamental concepts and main properties are listed in this section for the Hamiltonian realization under study.

Consider dynamic system

$\dot{x}=f(x), \quad x \in \mathscr{M}$,

where $\mathscr{M}$ is an $n$-dimensional manifold and $f(x)$ is an $n$ dimensional vector field with $f(0)=0$.

Definition 2.1 (Cheng et al. [4]). System (2.1) is said to have a Generalized Hamiltonian Realization (GHR) if there exists a 
suitable coordinate chart and a Hamiltonian function $H$ such that system (2.1) can be expressed as

$\dot{x}=T(x) \nabla H$,

where $\nabla H=\partial H / \partial x$. If the structure matrix $T(x)$ can be expressed as $T(x)=J(x)-R(x)$, with skew-symmetric $J(x)$ and positive semi-definite symmetric $R(x)$, then system (2.2) is called a DHR. Furthermore, if $R(x)>0,(2.2)$ is called a strictly DHR.

Definition 2.2 (Cheng et al. [4]). A controlled dynamic system

$\dot{x}=f(x)+g(x) u, \quad f(0)=0, \quad x \in \mathscr{M}, \quad u \in \mathbb{R}^{m}$

is said to have a state feedback Hamiltonian realization if there exists a suitable state feedback law $u=\alpha(x)+v$ such that the closed-loop system can be expressed as

$\dot{x}=T(x) \nabla H+g(x) v$.

If $T(x)$ can be expressed as $T(x)=J(x)-R(x)$, with $J(x)$ skew-symmetric and $R(x) \geqslant 0(>0)$, then (2.4) is called a feedback (strictly) DHR.

From Definition 2.1, it is easy to know that system (2.2) is a (strictly) dissipative realization if and only if $T(x)+$ $T^{\mathrm{T}}(x) \leqslant 0(<0)$.

Assume that $y=\phi(x)$ is a coordinate transformation. Under the new coordinate frame, the structure matrix $T(x)$ takes the form of the following: $T(y)=\left.J_{y}(x) T(x) J_{y}^{\mathrm{T}}(x)\right|_{x=\phi^{-1}(y)}$, where $J_{y}(x)$ is the Jacobian matrix of $y=\phi(x)$. Based on this, we know that the following result holds.

Proposition 2.3. The dissipativity of system (2.2) is invariant under coordinate transformations.

Definition 2.4 (Arnold [1]). Let $\lambda=\left(\lambda_{1}, \ldots, \lambda_{n}\right)$ be the eigenvalues of a given matrix $A \in \mathbb{R}^{n \times n}$. $A$ is said to be a resonant matrix if there exists $m=\left(m_{1}, \ldots, m_{n}\right) \in Z_{+}^{n}$, and $|m| \geqslant 2$, i.e., $m_{i} \geqslant 0$ and $\sum_{i=1}^{n} m_{i} \geqslant 2$, such that for some $s \in\{1,2, \ldots, n\}$,

$\lambda_{s}=\langle m, \lambda\rangle=m_{1} \lambda_{1}+\cdots+m_{n} \lambda_{n}$.

We call (2.5) the resonance condition, which is an essential concept for our further development.

Proposition 2.5 (Devanathan [8]). Let $\sigma(A)=\left\{\lambda_{1}, \ldots, \lambda_{n}\right\}$ be the eigenvalues of a given Hurwitz matrix $A \in \mathbb{R}^{n \times n}$. If

$\max \left\{\left|\operatorname{Re}\left(\lambda_{i}\right)\right|: \lambda_{i} \in \sigma(A)\right\} \leqslant 2 \min \left\{\left|\operatorname{Re}\left(\lambda_{i}\right)\right|: \lambda_{i} \in \sigma(A)\right\}$

holds, then A is non-resonant.

\section{Approximate DHR}

In general, it is hard to obtain the precise DHR form for nonlinear systems. When a precise DHR does not exist for a dynamic system or such a precise realization is difficulty to obtain, it is necessary to consider its approximate realization. This section investigates approximate DHR. We will propose the main result of the paper in this section, and establish a new algorithm for the realization. First, we present the concept of $k$ th degree approximate DHR.

\subsection{Basic concepts}

Definition 3.1. System (2.1) is said to have a $k$ th degree approximate DHR $(k \geqslant 1)$ if there exists a suitable coordinate chart and a Hamiltonian function $H$ such that system (2.1) can be expressed as

$\dot{x}=[J(x)-R(x)] \nabla H+\mathrm{O}\left(\|x\|^{k+1}\right)$,

where $J(x) \in \mathbb{R}^{n \times n}$ is skew-symmetric and $R(x) \in \mathbb{R}^{n \times n}$ is positive semi-definite.

Definition 3.2. System (2.3) is said to have a state feedback $k$ th degree approximate DHR $(k \geqslant 1)$ if there exists a suitable state feedback law $u=\alpha(x)+v$ such that the closed-loop system can be expressed as

$\dot{x}=[J(x)-R(x)] \nabla H+\mathrm{O}\left(\|x\|^{k+1}\right)+g(x) v$,

where $J(x) \in \mathbb{R}^{n \times n}$ is skew-symmetric, and $R(x) \in \mathbb{R}^{n \times n}$ is positive semi-definite.

From Definition 3.1, we obtain the following proposition.

Proposition 3.3. If the Hamiltonian function $H(x)$ has a local minimum at the origin, and if $R(x) \nabla H \sim \mathrm{O}\left(\|x\|^{l}\right)($ as $x \rightarrow 0)$, $0 \leqslant l \leqslant k$, then system (3.1) is locally stable.

Proof. Choosing $H(x)$ as the Lyapunov function, we obtain

$\dot{H}=-\mathrm{d} H R(x) \nabla H+\mathrm{d} H \cdot \mathrm{O}\left(\|x\|^{k+1}\right)$,

where $d H=\nabla^{\mathrm{T}} H$. Since $R(x) \nabla H \sim \mathrm{O}\left(\|x\|^{l}\right), l \leqslant k$, there exist a neighborhood, $\Omega$, of the origin such that $\dot{H} \leqslant 0$ in $\Omega$. Therefore, the approximate DHR (3.1) is locally stable.

\subsection{Main result}

This subsection studies $k$ th degree approximate DHR and proposes the main result of this paper. First, we present a lemma, which will be used in the derivation of the main result.

Given a constant matrix $A \in \mathbb{R}^{n \times n}$, for clarity we make the following assumption:

A1. Matrix $A$ is a constant diagonal matrix with distinct diagonal elements and is non-resonant.

With this $A$, define $L_{A x} X:=[A x, X]=(\partial X / \partial x) A x-A X$, where $x \in \mathbb{R}^{n},[\cdot, \cdot]$ is the Lie bracket, $X \in \mathbb{R}^{n}$ is a vector field, and $\partial X / \partial x$ is the Jacobian matrix of $X$. Then, we have the following lemma.

Lemma 3.4. Assume that matrix $A \in \mathbb{R}^{n \times n}$ satisfies $A 1$ and $\varphi(x)$ is a kth degree homogeneous vector field, $k \geqslant 2$. Then there 
exist a kth degree homogeneous vector field $\eta$ such that

$L_{A x} \eta=\varphi(x)$.

Proof. Assume that $\varphi(x)=\left[\varphi_{1}(x), \ldots, \varphi_{n}(x)\right]^{\mathrm{T}}$, where

$\varphi_{j}(x)=\sum_{i_{1}+\cdots+i_{n}=k} a_{i_{1} \cdots i_{n}}^{(j)} x_{1}^{i_{1}} \cdots x_{n}^{i_{n}}, \quad j=1,2, \ldots, n$.

Then, a straightforward computation shows that $L_{A x} \varphi(x)=$ $\left[\psi_{1}(x), \ldots, \psi_{n}(x)\right]^{\mathrm{T}}$, where

$\psi_{j}(x)=\sum_{i_{1}+\cdots+i_{n}=k} a_{i_{1} \cdots i_{n}}^{(j)} \cdot \mu_{i_{1} \cdots i_{n}}^{(j)} x_{1}^{i_{1}} \cdots x_{n}^{i_{n}}$,

$\mu_{i_{1} \cdots i_{n}}^{(j)}=i_{1} \lambda_{1}+\cdots+i_{n} \lambda_{n}-\lambda_{j}, \quad j=1,2, \ldots, n$.

Since $A$ is non-resonant, $\mu_{i_{1} \cdots i_{n}}^{(j)} \neq 0$. Define $\eta=\left[\eta_{1}, \ldots, \eta_{n}\right]^{\mathrm{T}}$, with

$\eta_{j}=\sum_{i_{1}+\cdots+i_{n}=k} \frac{a_{i_{1} \cdots i_{n}}^{(j)}}{\mu_{i_{1} \cdots i_{n}}^{(j)}} x_{1}^{i_{1}} \cdots x_{n}^{i_{n}}$.

Then, it follows that $L_{A x} \eta=\varphi(x)$.

Corollary 3.5. Assume that matrix $A \in \mathbb{R}^{n \times n}$ satisfies $A 1$ and a vector field $\varphi(x)=\mathrm{O}\left(\|x\|^{k}\right)$. Then, there exists a vector field $\eta=\mathrm{O}\left(\|x\|^{k}\right)$ such that $L_{A x} \eta=\varphi(x)$.

Proof. Expand $\varphi(x)$ as $\varphi(x)=\sum_{i \geqslant k} \varphi_{i} x^{i}$. Then, using Lemma 3.4 to each term can yield $\eta$.

Denote by $H_{k}$ the set of $k$ th degree homogeneous polynomial vector fields. Then $L_{A x}: H_{k} \rightarrow H_{k}$ is a linear map. Particularly, if $A$ is non-resonant, this map is surjective. Under the non-resonant assumption on $A$, the notation $L_{A x}^{-1}$ can be used, that is, $L_{A x}^{-1} g=\eta \Longleftrightarrow L_{A x} \eta=g$.

In the following, we study the $k$ th approximate DHR for system (2.3). In system (2.3), let $A:=J_{f}(0)$ and $B=g(0)$, where $J_{f}(x)$ is the Jacobian matrix of $f(x)$. We assume that $A$ and $B$ satisfy Assumption $\mathrm{A} 2$ :

A2. $(A, B)$ is a completely controllable pair.

Hereafter, we assume that all the vector fields and functions used in this paper are analytic, which implies that all the functions and their derivatives have convergent Taylor expansions.

Now, we are ready to present the main result of the paper.

Theorem 3.6. Assume that A2 holds for system (2.3). Then, for an arbitrary natural number $k \geqslant 1$, system (2.3) has a kth degree approximate DHR.

In the following, we apply the diagonal normal form in [5] to give a constructive proof for the theorem. The proof itself will provide a useful algorithm to find $k$ th degree approximate DHR for system (2.3).
Proof. Using Taylor expansion, system (2.3) can be expressed as

$\dot{x}=A x+\sum_{i \geqslant 2} F_{i} x^{i}+g(x) u$,

where $x^{i}=\underbrace{x \otimes x \otimes \cdots \otimes x}, i \geqslant 2, \otimes$ is the Kronecker product, and $F_{i}$ is an $n \times n^{i}$ matrix, $i=2,3, \ldots$.

Since Assumption A2 holds, without loss of generality, we can assume that matrix $A$ is a diagonal matrix with distinct diagonal elements $\lambda_{i}<0$ and is non-resonant (or else, a state feedback will do).

When $k=1$, let $R=-A$ and $H(x)=\frac{1}{2} \sum_{i=1}^{n} x_{i}^{2}$. Then, system (3.4) can be rewritten as

$\dot{x}=-R \nabla H+\mathrm{O}\left(\|x\|^{2}\right)+g u$,

which means that it holds for $k=1$.

When $k>1$, by setting

$z_{1}=x-L_{A x}^{-1}\left(F_{2} x^{2}\right)$,

system (3.4) can be expressed as (for convenience of analysis, let $u=0$ temporarily)

$$
\begin{aligned}
\dot{z}_{1}= & {\left[A x+\sum_{i \geqslant 2} F_{i} x^{i}\right]-\frac{\partial L_{A x}^{-1}\left(F_{2} x^{2}\right)}{\partial x}\left[A x+\sum_{i \geqslant 2} F_{i} x^{i}\right] } \\
= & -R \frac{\partial H_{1}}{\partial z_{1}}+\left[A L_{A x}^{-1}\left(F_{2} x^{2}\right)+F_{2} x^{2}-\frac{\partial L_{A x}^{-1}\left(F_{2} x^{2}\right)}{\partial x} A x\right] \\
& +\left[F_{3} x^{3}-\frac{\partial L_{A x}^{-1}\left(F_{2} x^{2}\right)}{\partial x} F_{2} x^{2}\right] \\
& +\left[F_{4} x^{4}-\frac{\partial L_{A x}^{-1}\left(F_{2} x^{2}\right)}{\partial x} F_{3} x^{3}\right]+\cdots \\
= & -R \frac{\partial H_{1}}{\partial z_{1}}+\left[F_{2} x^{2}-L_{A x}\left(L_{A x}^{-1}\left(F_{2} x^{2}\right)\right)\right] \\
& +\left[F_{3} x^{3}-\frac{\partial L_{A x}^{-1}\left(F_{2} x^{2}\right)}{\partial x} F_{2} x^{2}\right] \\
& +\left[F_{4} x^{4}-\frac{\partial L_{A x}^{-1}\left(F_{2} x^{2}\right)}{\partial x} F_{3} x^{3}\right]+\cdots \\
:= & -R \frac{\partial H_{1}}{\partial z_{1}}+\sum_{i \geqslant 3} F_{i}^{(1)} x^{i}=-R \frac{\partial H_{1}}{\partial z_{1}}+\mathrm{O}\left(\|x\|^{3}\right),
\end{aligned}
$$

where $H_{1}=\frac{1}{2} z_{1}^{\mathrm{T}} z_{1}$, and

$F_{i}^{(1)} x^{i}:=F_{i} x^{i}-\frac{\partial L_{A x}^{-1}\left(F_{2} x^{2}\right)}{\partial x} F_{i-1} x^{i-1}, \quad i \geqslant 3$.

It can be seen that system (3.7) is a second degree approximate DHR. If the approximation degree of (3.7) does not meet the requirement, i.e., $2<k$, then we go on to set

$z_{2}=z_{1}-L_{A x}^{-1}\left(F_{3}^{(1)} x^{3}\right)$. 
From (3.9), (3.7) and (3.4), we have

$$
\begin{aligned}
& \dot{z}_{2}=-R \frac{\partial H_{1}}{\partial z_{1}}+\sum_{i \geqslant 3} F_{i}^{(1)} x^{i}-\frac{\partial L_{A x}^{-1}\left(F_{3}^{(1)} x^{3}\right)}{\partial x} \\
& \times\left[A x+\sum_{i \geqslant 2} F_{i} x^{i}\right] \\
& =-R \frac{\partial H_{2}}{\partial z_{2}}+\left[R\left(\frac{\partial H_{2}}{\partial z_{2}}-\frac{\partial H_{1}}{\partial z_{1}}\right)+F_{3}^{(1)} x^{3}\right. \\
& \left.-\frac{\partial L_{A x}^{-1}\left(F_{3}^{(1)} x^{3}\right)}{\partial x} A x\right] \\
& +\left[F_{4}^{(1)} x^{4}-\frac{\partial L_{A x}^{-1}\left(F_{3}^{(1)} x^{3}\right)}{\partial x} F_{2} x^{2}\right] \\
& +\left[F_{5}^{(1)} x^{5}-\frac{\partial L_{A x}^{-1}\left(F_{3}^{(1)} x^{3}\right)}{\partial x} F_{3} x^{3}\right]+\cdots \\
& :=-R \frac{\partial H_{2}}{\partial z_{2}}+\sum_{i \geqslant 4} F_{i}^{(2)} x^{i}=-R \frac{\partial H_{2}}{\partial z_{2}}+\mathrm{O}\left(\|x\|^{4}\right) \text {, }
\end{aligned}
$$

where $H_{2}=\frac{1}{2} z_{2}^{\mathrm{T}} z_{2}$, and

$F_{i}^{(2)} x^{i}:=F_{i}^{(1)} x^{i}-\frac{\partial L_{A x}^{-1}\left(F_{3}^{(1)} x^{3}\right)}{\partial x} F_{i-2} x^{i-2}, \quad i \geqslant 4$.

Recursively, setting

$z_{k}=z_{k-1}-L_{A x}^{-1}\left(F_{k+1}^{(k-1)} x^{k+1}\right)$,

we obtain

$$
\begin{aligned}
\dot{z}_{k} & =-R \frac{\partial H_{k}}{\partial z_{k}}+F_{k+2}^{(k)} x^{k+2}+F_{k+3}^{(k)} x^{k+3}+\cdots \\
& =-R \frac{\partial H_{k}}{\partial z_{k}}+\mathrm{O}\left(\|x\|^{k+2}\right),
\end{aligned}
$$

where

$$
\begin{aligned}
& F_{i}^{(k)} x^{i}:=F_{i}^{(k-1)} x^{i}-\frac{\partial L_{A x}^{-1}\left(F_{k+1}^{(k-1)} x^{k+1}\right)}{\partial x} F_{i-k} x^{i-k}, \\
& \quad i \geqslant k+2,
\end{aligned}
$$

$z_{0}:=x, \quad F_{i}^{(0)}:=F_{i}, \quad H_{k}=\frac{1}{2} z_{k}^{\mathrm{T}} z_{k}, \quad k=1,2, \ldots$.

Using (3.12) repeatedly, we have

$z_{k}=x-\sum_{i=2}^{k+1} L_{A x}^{-1}\left(F_{i}^{(i-2)} x^{i}\right):=\psi_{k}(x)$

Because $\left(\partial \psi_{k} / x\right)(0)=I_{n}$, Eq. (3.15) can serve as a coordinate transformation in some neighborhood, $\Omega$, of the origin.
From (3.15) and (3.13), we obtain

$$
\begin{aligned}
\dot{z}_{k} & =\frac{\partial \psi_{k}}{\partial x} \dot{x}=-R \frac{\partial H_{k}}{\partial z_{k}}+\mathrm{O}\left(\|x\|^{k+2}\right) \\
& =-R\left(\frac{\partial \psi_{k}^{-1}}{\partial z_{k}}\right)^{\mathrm{T}} \frac{\partial H_{k}(x)}{\partial x}+\mathrm{O}\left(\|x\|^{k+2}\right),
\end{aligned}
$$

which implies

$$
\begin{aligned}
\dot{x}= & {\left[-\left(\frac{\partial \psi_{k}}{\partial x}\right)^{-1} R\left(\frac{\partial \psi_{k}}{\partial x}\right)^{-\mathrm{T}}\right] \frac{\partial H_{k}(x)}{\partial x}+\left(\frac{\partial \psi_{k}}{\partial x}\right)^{-1} } \\
& \times \mathrm{O}\left(\|x\|^{k+2}\right) .
\end{aligned}
$$

Thus, system (2.3) has a $k$ th degree approximate DHR as follows:

$\dot{x}=-R_{k-1} \frac{\partial H_{k-1}(x)}{\partial x}+\mathrm{O}\left(\|x\|^{k+1}\right)+g(x) u$,

where

$$
\begin{aligned}
R_{k-1}= & \left(\frac{\partial \psi_{k-1}}{\partial x}\right)^{-1} R\left(\frac{\partial \psi_{k-1}}{\partial x}\right)^{-\mathrm{T}}>0, \\
H_{k-1}(x)= & \frac{1}{2} \psi_{k-1}^{\mathrm{T}}(x) \psi_{k-1}(x) \\
= & \frac{1}{2}\left(x-\sum_{i=2}^{k} L_{A x}^{-1}\left(F_{i}^{(i-2)} x_{i}\right)\right)^{\mathrm{T}} \\
& \times\left(x-\sum_{i=2}^{k} L_{A x}^{-1}\left(F_{i}^{(i-2)} x_{i}\right)\right)^{k}
\end{aligned}
$$

Based on the proof of Theorem 3.6, we can establish the following algorithm of finding $k$ th degree approximate DHR for system (2.3).

Algorithm 3.7. Assume that A2 holds for system (2.3). Then, the $k$ th degree approximate DHR of system (2.3) can be obtained in four steps as follows $(k \geqslant 2)$ :

Step 1: Express system (2.3) as (3.4), such that $A=$ $\operatorname{Diag}\left\{\lambda_{1}, \ldots, \lambda_{n}\right\}<0$ satisfies Assumption $A 1$.

Step 2: Find $\eta_{i}:=L_{A x}^{-1}\left(F_{i}^{(i-2)} x^{i}\right), i=2,3, \ldots, k$, where $F_{i}^{(0)}=F_{i}$.

From the proof of Lemma 3.4, we know

$\eta_{2}=L_{A x}^{-1}\left(F_{2} x^{2}\right)$

$$
\begin{aligned}
= & {\left[\begin{array}{ccccc}
\frac{1}{2 \lambda_{1}-\lambda_{1}} & \frac{1}{\lambda_{1}+\lambda_{2}-\lambda_{1}} & \cdots & \frac{1}{\lambda_{n}+\lambda_{n-1}-\lambda_{1}} & \frac{1}{2 \lambda_{n}-\lambda_{1}} \\
\frac{1}{2 \lambda_{1}-\lambda_{2}} & \frac{1}{\lambda_{1}+\lambda_{2}-\lambda_{2}} & \cdots & \frac{1}{\lambda_{n}+\lambda_{n-1}-\lambda_{2}} & \frac{1}{2 \lambda_{n}-\lambda_{2}} \\
& \cdots & & \cdots & \\
\frac{1}{2 \lambda_{1}-\lambda_{n}} & \frac{1}{\lambda_{1}+\lambda_{2}-\lambda_{n}} & \cdots & \frac{1}{\lambda_{n}+\lambda_{n-1}-\lambda_{n}} & \frac{1}{2 \lambda_{n}-\lambda_{n}}
\end{array}\right]_{n \times n^{2}} } \\
\odot F_{2} x^{2} &
\end{aligned}
$$


where $\odot$ is the Hadamard product of matrices [15], which is defined as follows. Assume that $P=\left[p_{i j}\right] \in \mathbb{R}^{m \times n}, Q=\left[q_{i j}\right] \in$ $\mathbb{R}^{m \times n}$ are two matrices. Then, $P \odot Q$ is defined as $P \odot Q=$ $\left[p_{i j} q_{i j}\right]_{m \times n}$. Using (3.14) repeatedly yields

$F_{i}^{(i-2)} x^{i}=F_{i} x^{i}-\sum_{s=1}^{i-2} \frac{\partial \eta_{s+1}}{\partial x} F_{i-s} x^{i-s}$,

$i=3,4, \ldots, k$.

From the proof of Lemma 3.4 again, we have

$$
\begin{aligned}
\eta_{i}= & L_{A x}^{-1}\left(F_{i}^{(i-2)} x^{i}\right) \\
= & {\left[\begin{array}{ccccc}
\frac{1}{i \lambda_{1}-\lambda_{1}} & \frac{1}{(i-1) \lambda_{1}+\lambda_{2}-\lambda_{1}} & \cdots & \frac{1}{(i-1) \lambda_{n}+\lambda_{n-1}-\lambda_{1}} & \frac{1}{i \lambda_{n}-\lambda_{1}} \\
\frac{1}{i \lambda_{1}-\lambda_{2}} & \frac{1}{(i-1) \lambda_{1}+\lambda_{2}-\lambda_{2}} & \cdots & \frac{1}{(i-1) \lambda_{n}+\lambda_{n-1}-\lambda_{2}} & \frac{1}{i \lambda_{n}-\lambda_{2}} \\
\cdots & \cdots & & \cdots & \\
\frac{1}{i \lambda_{1}-\lambda_{n}} & \frac{1}{(i-1) \lambda_{1}+\lambda_{2}-\lambda_{n}} & \cdots & \frac{1}{(i-1) \lambda_{n}+\lambda_{n-1}-\lambda_{n}} & \frac{1}{i \lambda_{n}-\lambda_{n}}
\end{array}\right]_{n \times n^{i}} } \\
& \odot F_{i}^{(i-2)} x^{i}, i=3,4, \ldots, k .
\end{aligned}
$$

Eqs. (3.19)-(3.21) are the recursive formula of finding $\eta_{i}$.

Step 3: Find the coordinate transformation $z_{k-1}=\psi_{k-1}(x)$.

Using (3.15), we obtain

$z_{k-1}=\psi_{k-1}(x)=x-\sum_{i=2}^{k} \eta_{i}$.

Step 4. Calculate $R_{k-1}=-\left(\partial \psi_{k-1} / \partial x\right)^{-1} A\left(\partial \psi_{k-1} / \partial x\right)^{-\mathrm{T}}$ $>0, H_{k-1}=\frac{1}{2} \psi_{k-1}^{\mathrm{T}}(x) \psi_{k-1}(x)$, and give the form of $k$ th degree approximate DHR as follows:

$\dot{x}=-R_{k-1} \frac{\partial H_{k-1}}{\partial x}+\mathrm{O}\left(\|x\|^{k+1}\right)+g(x) u$.

Remark 3.8. In Step 1 , if $A$ does not satisfy Assumption $A 1$, we can add a state feedback law to system (2.3) such that Assumption $A 1$ holds for the system. This can be guaranteed by Assumption A2.

Remark 3.9. It is easy to see that $H_{k-1}$ has a strictly local minimum at the origin, and the degree of the lowest-degree nonzero terms in $-R_{k-1} \nabla H_{k-1}$ is no more than $k$. From Proposition 3.3, the $k$ th degree approximate DHR (3.23) is locally stable (when $u=0$ ).

In Section 5, we will give an example to show how to apply Algorithm 3.7 to find a $k$ th degree approximate DHR.

Now, let $k \rightarrow \infty$ in (3.15), then we obtain

$z=x-\sum_{i=2}^{\infty} \eta_{i}:=\psi(x)$

Since $\psi(0)=0$, the convergent domain of (3.24), denoted by $\Theta$, is not empty, with which we have the following result.
Corollary 3.10. If $\Theta$ contains a neighborhood, $\Omega$, of the origin, then system (2.3) has a DHR as follows:

$\dot{x}=-\bar{R} \frac{\partial H}{\partial x}+g(x) u, \quad x \in \Omega$,

where

$\bar{R}=-\left(\frac{\partial \psi}{\partial x}\right)^{-1} A\left(\frac{\partial \psi}{\partial x}\right)^{-\mathrm{T}}>0$

and

$H=\frac{1}{2} \psi^{\mathrm{T}}(x) \psi(x)=\frac{1}{2}\left(x-\sum_{i=2}^{\infty} \eta_{i}\right)^{\mathrm{T}}\left(x-\sum_{i=2}^{\infty} \eta_{i}\right)$.

In system (2.3), if $f(x)$ is a polynomial vector field, we can obtain a much more elegant result as follows.

Corollary 3.11. If $f(x)$ is a kth degree polynomial vector field, then system (2.3) has the following DHR:

$\dot{x}=-\bar{R} \frac{\partial H}{\partial x}+g(x) u$,

where

$\bar{R}=-\left(\frac{\partial \psi_{k-1}}{\partial x}\right)^{-1} A\left(\frac{\partial \psi_{k-1}}{\partial x}\right)^{-\mathrm{T}}>0$

and

$H=\frac{1}{2} \psi_{k-1}^{\mathrm{T}}(x) \psi_{k-1}(x)=\frac{1}{2}\left(x-\sum_{i=2}^{k} \eta_{i}\right)^{\mathrm{T}}\left(x-\sum_{i=2}^{k} \eta_{i}\right)$.

Corollary 3.11 shows that every polynomial system has a precise DHR, which can be given by (3.26).

\section{4. $k$ th degree approximate Lyapunov function}

This section investigates construction of local Lyapunov functions. First, we propose the concept of $k$ th degree approximate Lyapunov function, and then, based on the approximate DHR algorithm obtained in Section 3, we provide a method of constructing the $k$ th degree approximate Lyapunov function.

Consider system

$\dot{x}=f(x), \quad f(0)=0, \quad x \in \mathbb{R}^{n}$.

Definition 4.1. A scalar function $V(x)$ is called a $k$ th degree approximate Lyapunov function of system (4.1) if

(1) $V(x)$ is positive definite; and

(2) $\dot{V}(x)+\mathrm{O}\left(\|x\|^{k+1}\right)=\delta_{k}(x) \leqslant 0$ holds along the trajectory of system (4.1), where $\delta_{k}(x) \sim \mathrm{O}\left(\|x\|^{l}\right)$ (as $x \rightarrow$ $0), 0 \leqslant l \leqslant k$.

Example 4.2. $V(x)=\frac{1}{2} x_{1}^{2}+x_{2}^{2}$ is a second degree approximate Lyapunov function of the following system:

$\left[\begin{array}{c}\dot{x}_{1} \\ \dot{x}_{2}\end{array}\right]=\left[\begin{array}{c}-x_{1}+x_{2}^{2} \\ -x_{2}+x_{1}^{3}\end{array}\right]:=f(x)$. 
In fact,

$$
\begin{aligned}
\dot{V}(x) & =L_{f} V(x)=-x_{1}^{2}-2 x_{2}^{2}+x_{1} x_{2}^{2}+2 x_{1}^{3} x_{2} \\
& :=\delta_{2}(x)+\mathrm{O}\left(\|x\|^{3}\right),
\end{aligned}
$$

where $\delta_{2}(x)=-x_{1}^{2}-2 x_{2}^{2}$ is negative definite and $\delta_{2}(x) \sim$ $\mathrm{O}\left(\|x\|^{2}\right)$. From Definition 4.1, $V(x)$ is a second degree approximate Lyapunov function of system (4.2).

From Definition 4.1, it can be seen that the following result holds.

Proposition 4.3. If $V(x)$ is a kth degree approximate Lyapunov function of system (4.1), then $V(x)$ is a local Lyapunov function of the system.

Next, we present a method of constructing $k$ th degree approximate Lyapunov function for system (2.3).

Proposition 4.4. Algorithm 3.7 provides an algorithm to obtain kth degree approximate Lyapunov function for system (2.3).

Proof. Consider the $k$ th degree approximate DHR (3.23). It is easy to see that $H_{k-1}(x)=\frac{1}{2} \psi_{k-1}^{\mathrm{T}} \psi_{k-1}$ is positive definite. When $u=0$,

$$
\begin{aligned}
\dot{H}_{k-1}= & \left(\frac{\partial H_{k-1}}{\partial x}\right)^{\mathrm{T}}\left(-R_{k-1} \frac{\partial H_{k-1}}{\partial x}+\mathrm{O}\left(\|x\|^{k+1}\right)\right) \\
= & \psi_{k-1}^{\mathrm{T}} \frac{\partial \psi_{k-1}}{\partial x}\left[\left(\frac{\partial \psi_{k-1}}{\partial x}\right)^{-1} A\left(\frac{\partial \psi_{k-1}}{\partial x}\right)^{-\mathrm{T}}\right] \\
& \times\left(\frac{\partial \psi_{k-1}}{\partial x}\right)^{\mathrm{T}} \psi_{k-1}+\psi_{k-1}^{\mathrm{T}} \frac{\partial \psi_{k-1}}{\partial x} \cdot \mathrm{O}\left(\|x\|^{k+1}\right) \\
= & \psi_{k-1}^{\mathrm{T}} A \psi_{k-1}+\mathrm{O}\left(\|x\|^{k+2}\right):=\delta_{k+1}(x)+\mathrm{O}\left(\|x\|^{k+2}\right),
\end{aligned}
$$

where $\delta_{k+1}(x)=\psi_{k-1}^{\mathrm{T}} A \psi_{k-1}$ is negative definite and $\delta_{k+1}(x) \sim \mathrm{O}\left(\|x\|^{2}\right)($ as $x \rightarrow 0)$. From Definition 4.1, we know that $H_{k-1}$ is a $(k+1)$ th degree approximate Lyapunov function of system (2.3).

Remark 4.5. When using Algorithm 3.7 to find $s$ th degree approximate Lyapunov function $(s \geqslant 2)$, we should let $k=s-1$ in Steps 2-4 and find $H_{s-2}$, which is the desired sth degree approximate Lyapunov function.

Remark 4.6. With the method presented in this paper, we can easily obtain an explicit DHR form (approximate form) as well as a kind of local Lyapunov function for the system whose linearization is controllable. Once the DHR form is obtained, all the existing results on control design based on Hamiltonian systems in the literature can be used for the system under consideration. This is yet another objective of the paper to bridge the gap of existing energy-based control design results for this class of systems by providing explicit algorithms to construct the DHR, and thus bringing the related methods more close to reality.

\section{An illustrative example}

In this section, we give an example, which cannot be provided a DHR by using the results in [29,23], to show how to apply Algorithm 3.7 to find $k$ th degree approximate DHR and $k$ th degree approximate Lyapunov function for nonlinear systems.

Example 5.1. Find a fourth degree approximate DHR and a fifth degree approximate Lyapunov function for the following system:

$\dot{x}=\left[\begin{array}{c}1-e^{3 x_{1}} \\ -x_{1}^{2}-4 x_{2}-x_{3}^{2} \\ x_{1}^{3}+x_{3}\end{array}\right]+\left[\begin{array}{l}0 \\ 0 \\ 1\end{array}\right] u$

$:=f(x)+g u, x \in \mathbb{R}^{3}$

It is easy to see that $f(x)$ is an analytic vector field. A straightforward computation shows that $J_{f}(0)=\operatorname{Diag}\{-3$, $-4,1\}$ and $g(0)=[0,0,1]^{\mathrm{T}}$. It can be checked that $\left(J_{f}(0), g(0)\right)$ is a controllable pair. Notice that $J_{f}(0)$ does not satisfy Assumption A1. We first choose a state feedback

$u=-6 x_{3}+v$

and substitute it into (5.1). Then, we have

$\dot{x}=\left[\begin{array}{c}1-e^{3 x_{1}} \\ -x_{1}^{2}-4 x_{2}-x_{3}^{2} \\ x_{1}^{3}-5 x_{3}\end{array}\right]+\left[\begin{array}{l}0 \\ 0 \\ 1\end{array}\right] v$.

In the example, $k=4$. Using Taylor expansion, we express system (5.3) as follows:

$\dot{x}=A x+F_{2} x^{2}+F_{3} x^{3}+F_{4} x^{4}+\mathrm{O}\left(\|x\|^{5}\right)+g v$,

where

$A=\left[\begin{array}{ccc}-3 & 0 & 0 \\ 0 & -4 & 0 \\ 0 & 0 & -5\end{array}\right]$,

$F_{2}=\left[\begin{array}{ccccccccc}-\frac{9}{2} & 0 & 0 & 0 & 0 & 0 & 0 & 0 & 0 \\ -1 & 0 & 0 & 0 & 0 & 0 & 0 & 0 & -1 \\ 0 & 0 & 0 & 0 & 0 & 0 & 0 & 0 & 0\end{array}\right]$,

$F_{3}=\left[\begin{array}{cccc}-\frac{27}{3} & 0 & \cdots & 0 \\ 0 & 0 & \cdots & 0 \\ 1 & 0 & \cdots & 0\end{array}\right]_{3 \times 27}$,

$F_{4}=\left[\begin{array}{cccc}-\frac{27}{8} & 0 & \cdots & 0 \\ 0 & 0 & \cdots & 0 \\ 0 & 0 & \cdots & 0\end{array}\right]_{3 \times 81}$,

$x^{2}=\left[x_{1}^{2}, x_{1} x_{2}, x_{1} x_{3}, \ldots, x_{3} x_{2}, x_{3}^{2}\right]^{\mathrm{T}}, x^{3}=\left[x_{1}^{3}, x_{1}^{2} x_{2}, x_{1}^{2} x_{3}\right.$,

$\left.\ldots, x_{3}^{2} x_{2}, x_{3}^{3}\right]^{\mathrm{T}}$ and $x^{4}=\left[x_{1}^{4}, x_{1}^{3} x_{2}, x_{1}^{3} x_{3}, \ldots, x_{3}^{3} x_{2}, x_{3}^{4}\right]^{\mathrm{T}}$. 
It is easy to see from Proposition 2.5 that Assumption A1 holds for the system. Now, we find $\eta_{i}, i=2,3,4$. Since $F_{2} x^{2}=$ $\left[-\frac{9}{2} x_{1}^{2},-x_{1}^{2}-x_{3}^{2}, 0\right]^{\mathrm{T}}$, from (3.19) we obtain

$\eta_{2}=L_{A x}^{-1}\left(F_{2} x^{2}\right)=\left[\frac{3}{2} x_{1}^{2}, \frac{1}{2} x_{1}^{2}+\frac{1}{6} x_{3}^{2}, 0\right]^{\mathrm{T}}$.

From (3.20) and (3.21), we have

$$
\begin{gathered}
F_{3}^{(1)} x^{3}=F_{3} x^{3}-\frac{\partial \eta_{2}}{\partial x} F_{2} x^{2} \\
=\left[9 x_{1}^{3}, \frac{9}{2} x_{1}^{3}+\frac{1}{3} x_{1}^{2} x_{3}+\frac{1}{3} x_{3}^{3}, x_{1}^{3}\right]^{\mathrm{T}}, \\
\eta_{3}=L_{A x}^{-1}\left(F_{3}^{(1)} x^{3}\right) \\
=\left[-\frac{3}{2} x_{1}^{3},-\frac{9}{10} x_{1}^{3}-\frac{1}{21} x_{1}^{2} x_{3}-\frac{1}{33} x_{3}^{3},-\frac{1}{4} x_{1}^{3}\right]^{\mathrm{T}} .
\end{gathered}
$$

From (3.20) and (3.21) again,

$$
\begin{gathered}
F_{4}^{(2)} x^{4}=F_{4} x^{4}-\frac{\partial \eta_{2}}{\partial x} F_{3} x^{3}-\frac{\partial \eta_{3}}{\partial x} F_{2} x^{2} \\
=\left[-\frac{81}{8} x_{1}^{4},-\frac{153}{20} x_{1}^{4}-\frac{16}{21} x_{1}^{3} x_{3},-\frac{27}{8} x_{1}^{4}\right]^{\mathrm{T}}, \\
\eta_{4}=L_{A x}^{-1}\left(F_{4}^{(2)} x^{4}\right)=\left[\frac{9}{8} x_{1}^{4}, \frac{153}{160} x_{1}^{4}+\frac{8}{105} x_{1}^{3} x_{3}, \frac{27}{56} x_{1}^{4}\right]^{\mathrm{T}} .
\end{gathered}
$$

By (3.22), we obtain the desired coordinate transformation as follows:

$$
\begin{aligned}
z_{3} & =\psi_{3}(x)=x-\sum_{i=2}^{4} \eta_{i} \\
& =\left[\begin{array}{c}
x_{1}-\frac{3}{2} x_{1}^{2}+\frac{3}{2} x_{1}^{3}-\frac{9}{8} x_{1}^{4} \\
x_{2}-\frac{1}{2} x_{1}^{2}-\frac{1}{6} x_{3}^{2}+\frac{9}{10} x_{1}^{3}+\frac{1}{21} x_{1}^{2} x_{3}+\frac{1}{33} x_{3}^{3}-\frac{153}{160} x_{1}^{4}-\frac{8}{105} x_{1}^{3} x_{3} \\
x_{3}+\frac{1}{4} x_{1}^{3}-\frac{27}{56} x_{1}^{4}
\end{array}\right] .
\end{aligned}
$$

Thus, according to (3.23), system (5.1) has the following fourth degree approximate DHR:

$\dot{x}=-R_{3} \frac{\partial H_{3}}{\partial x}+g v+\mathrm{O}\left(\|x\|^{5}\right)$,

where

$$
\begin{aligned}
& R_{3}=-\left(\frac{\partial \psi_{3}}{\partial x}\right)^{-1} A\left(\frac{\partial \psi_{3}}{\partial x}\right)^{-\mathrm{T}}>0, \\
& H_{3}(x)=\frac{1}{2} \psi_{3}^{\mathrm{T}}(x) \psi_{3}(x) .
\end{aligned}
$$

Moreover, from Proposition 4.4, the Hamiltonian function $H_{3}(x)$ is just the desired fifth degree approximate Lyapunov function of system (5.1).

\section{Conclusion}

Using the diagonal normal form of dynamic systems, we have investigated the $k$ th degree approximate DHR of nonlinear systems and set up a new algorithm for the realization. It was shown that every nonlinear affine system has an approximate DHR if linearization of the system is controllable. We have also given the concept of $k$ th degree approximate Lyapunov function and proposed a method of constructing the approximate Lyapunov function. The presented example shows that the algorithm proposed in this paper is very feasible.

\section{References}

[1] V.I. Arnold, Geometrical Methods on the Theory of Ordinary Differential Equations, Springer, New York, 1983, pp. 177-188.

[2] F. Bullo, Averaging and vibrational control of mechanical systems, SIAM J. Control Optim. 41 (2) (2002) 542-562.

[3] D. Cheng, Z. Xi, Y. Hong, H. Qin, Energy-based stabilization in power systems, Proceedings of the 14th IFAC World Congress, Beijing, China, vol. O, 1999, pp. 297-303.

[4] D. Cheng, S. Spurgeon, J. Xiang, On the development of generalized Hamiltonian realizations, Proceedings of the 39th IEEE Conference on Decision and Control, Sydney, Australia, vol. 5, 2000, pp. 5125-5130.

[5] D. Cheng, X. Hu, Y. Wang, Non-regular feedback linearization of nonlinear systems via a normal form algorithm, Automatica 40 (2004) 439-447.

[6] T.J. Courant, Dirac manifold, Trans. Amer. Math. Soc. 319 (1990) 631-661.

[7] M. Dalsmo, A.J. van der Schaft, On representations and integrability of mathematical structures in energy-conserving physical systems, SIAM J. Control Optim. 37 (1) (1999) 54-91.

[8] R. Devanathan, Linearization condition through state feedback, IEEE Trans. Automat. Control 46 (8) (2001) 1257-1260.

[9] G. Escobar, A.J. van der Schaft, R. Ortega, A Hamiltonian viewpoint in the modeling of switching power converters, Automatica 35 (3) (1999) 445-452.

[10] I. Fantoni, R. Lozano, M.W. Spong, Energy based control of the pendubot, IEEE Trans. Automat. Control 45 (4) (2000) 725-729.

[11] T.I. Fossen, Marine Control Systems: Guidance, Navigation, and Control of Ships, Rigs and Underwater Vehicles, Marine Cybernetics AS, Trondheim, Norway, 2002.

[12] K. Fujimoto, T. Sugie, Canonical transformations and stabilization of generalized Hamiltonian systems, Systems Control Lett. 42 (2001) 217-227.

[13] K. Fujimoto, T. Sugie, Stabilization of Hamiltonian systems with nonholonomic constraints based on time-varying generalized canonical transformations, Systems control Lett. 44 (2001) 309-319.

[14] M. Galaz, R. Ortega, A.S. Bazanella, A.M. Stankovic, An energy-shaping approach to the design of excitation control of synchronous generators, Automatica 39 (1) (2003) 111-119.

[15] S.S. Ge, T.H. Lee, C.J. Harris, Adaptive Neural Network Control of Robotic Manipulators, World Scientific, London, 1998.

[16] H. Khalil, Nonlinear Systems, second ed., Prentice-Hall, New Jersey, 1996.

[17] A. Macchelli, C. Melchiorri, Modeling and control of the Timoshenko beam: the distributed port Hamiltonian approach, SIAM J. Control Optim. 43 (2) (2004) 743-767.

[18] B.M. Maschke, A.J. van der Schaft, Port-controlled Hamiltonian systems: modeling origins and system theoretic properties, Proceedings of the IFAC symposium on NOLCOS, Bordeaux, France, 1992, pp. 282-288.

[19] B.M. Maschke, R. Ortega, A.J. van der Schaft, Energy-based Lyapunov functions for forced Hamiltonian systems with dissipation, IEEE Trans. Automat. Control 45 (8) (2000) 1498-1502.

[20] L. Moreau, D. Aeyels, A novel variational method for deriving Lagrangian and Hamiltonian models of inductor-capacitor circuits, SIAM Rev. 46 (1) (2004) 59-84. 
[21] H. Nijmeijer, A.J. van der Schaft, Nonlinear Dynamical Control Systems, Springer, Berlin, 1990.

[22] R. Ortega, A. Loría, P.J. Nicklasson, H. Sira-Ramírez, Passivity-based Control of Euler-Lagrangian Systems. Communications and Control Engineering, Springer, Berlin, 1998.

[23] R. Ortega, A.J. van der Schaft, B. Maschke, G. Escobar, Interconnection and damping assignment passivity-based control of port-controlled Hamiltonian systems, Automatica 38 (4) (2002) 585-596.

[24] T. Shen, R. Ortega, Q. Lu, S. Mei, K. Tamura, Adaptive $L_{2}$ disturbance attenuation of Hamiltonian systems with parameter perturbations and application to power systems, Proceedings of the 39th IEEE Conference on Decision and Control, vol. 5, 2000, pp. 4939-4944.

[25] H. Sira-Ramírez, A general canonical form of feedback passivity of nonlinear systems, Internat. J. Control 71 (5) (1998) 891-905.

[26] J.E. Slotine, W. Li, Applied Nonlinear Control, Prentice-Hall, Englewood Cliffs, NJ, 1991
[27] S. Stramigioli, Modeling and IPC Control of Interactive Mechanical Systems: A Coordinate-free Approach, Springer, London, 2001.

[28] Y. Wang, D. Cheng, C. Li, Y. Ge, Dissipative Hamiltonian realization and energy-based $L_{2}$-disturbance attenuation control of multimachine power systems, IEEE Trans. Automat. Control 48 (8) (2003) 1428-1433.

[29] Y. Wang, C. Li, D. Cheng, Generalized Hamiltonian realization of timeinvariant nonlinear systems, Automatica 39 (8) (2003) 1437-1443.

[30] Y. Wang, C. Li, D. Cheng, New approaches to generalized Hamiltonian realization of nonlinear systems, Sci. in China Ser. F 46 (6) (2003) 431-444.

[31] A.J. van der Schaft, $L_{2}$-gain and Passivity Techniques in Nonlinear Control, Springer, Berlin, 1999.

[32] A.J. van der Schaft, B.M. Maschke, The Hamiltonian formulation of energy conserving physical systems with external ports, Archive für Elektronik und Übertragungstechnik 49 (1995) 362-371. 